\title{
Gastric bezoars
}

\author{
Sabiye Akbulut ${ }^{1}$, Kamuran Cumhur Değer ${ }^{2}$, Mustafa Duman², Sinan Yol ${ }^{2}$
}

${ }^{1}$ Department of Gastroenterology, Kartal Koşuyolu High Specialty Education and Research Hospital, Istanbul, Turkey

${ }^{2}$ Department of Gastroenterology Surgery, Kartal Koşuyolu High Specialty Education and Research Hospital, Istanbul, Turkey

Prz Gastroenterol 2016; 11 (1): 60-61

DOI: $10.5114 /$ pg.2015.55188

Address for correspondence: Kamuran Cumhur Değer, Department of Gastroenterology Surgery, Kartal Koşuyolu High Specialty Education and Research Hospital, 34860 Istanbul, Turkey, phone: +90 5334556478, e-mail: cumhurdeger@hotmail.com

Bezoar is the accumulation and mass formation of indigestible materials inside the gastrointestinal tract on account of several factors. Bezoars are relatively infrequent, with a reported incidence of $0.07 \%$ to $0.4 \%$ [1]. Bezoars are classified into several types according to their composition: plant and vegetable fibres (phytobezoars), hair (trichobezoars), medications (pharmacobezoars), or milk protein in milk-fed infants (lactobezoars). Elderly patients and those with diabetes mellitus or a history of gastrointestinal surgery are more likely to develop bezoars due to impaired gastric motility and delayed gastric emptying. Additionally, mental retardation and trichotillomania also are known as risk factors for the development of trichobezoars. Bezoars are mostly seen in stomach, oesophagus, small bowel, large bowel, and bile duct. It can be presented with anaemia, haematemesis, nausea, vomiting, and weight loss. Iron deficiency anaemia, which is widely common in patients with trichobezoar, is mostly a direct outcome

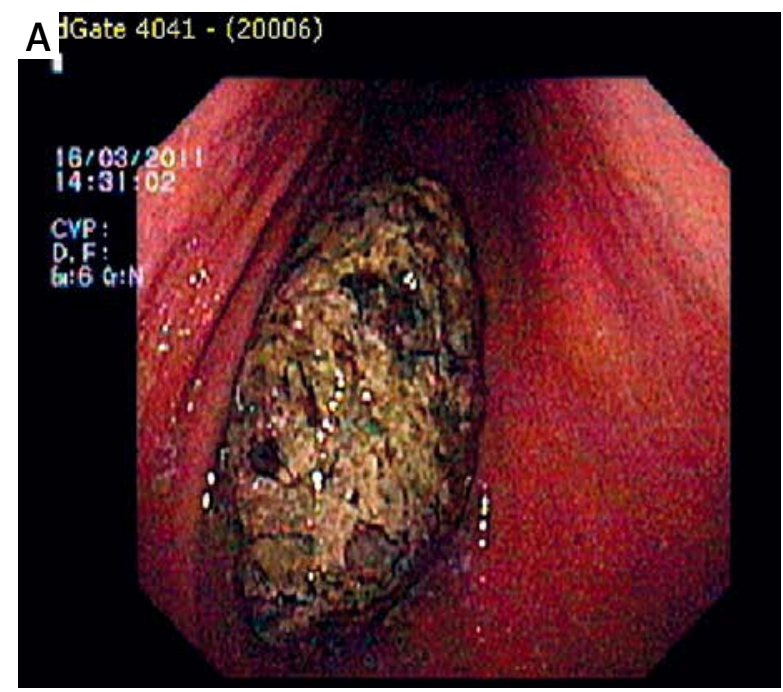

Figure $1 \mathrm{~A}, \mathrm{~B}$. Endoscopic view of the bezoar of some gastric ulcers caused by the pressure effect of mass. Bezoars can cause gastrointestinal complications like bleeding, gastric outlet obstruction, and ileus [2]. In this case we present an endoscopic removal of bezoar from a mentally retarded female patient.

A mentally retarded 51-year-old female patient was admitted to our hospital for symptoms of epigastric pain, nausea, vomiting, and weight loss (10 kg in the preceding year) over the course of the previous 3 years and aggravated in the previous month. She had a hair- and nail-eating habitat. In her blood samples, haemoglobin was $7.8 \mathrm{~g} / \mathrm{dl}$ and mean corpuscular volume (MCV) was $61.2 \mathrm{fl}$. Two hard black/yellowish coloured bezoars were revealed in her upper gastrointestinal endoscopy (Figures $1 \mathrm{~A}-\mathrm{B}$ ). Bezoars were fractionated by polypectomy snare catheter and extracted in two sessions. There were two ulcers of size 3-4 $\mathrm{mm}$ in diameter seen after complete removal of the bezoars (Figure 2). The patient's symptoms were relieved after this endoscopic intervention.

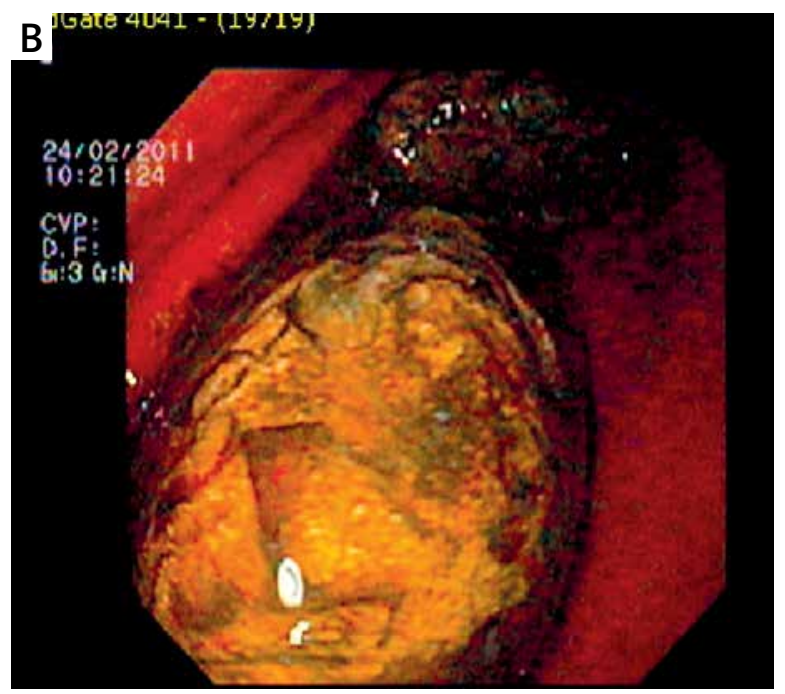




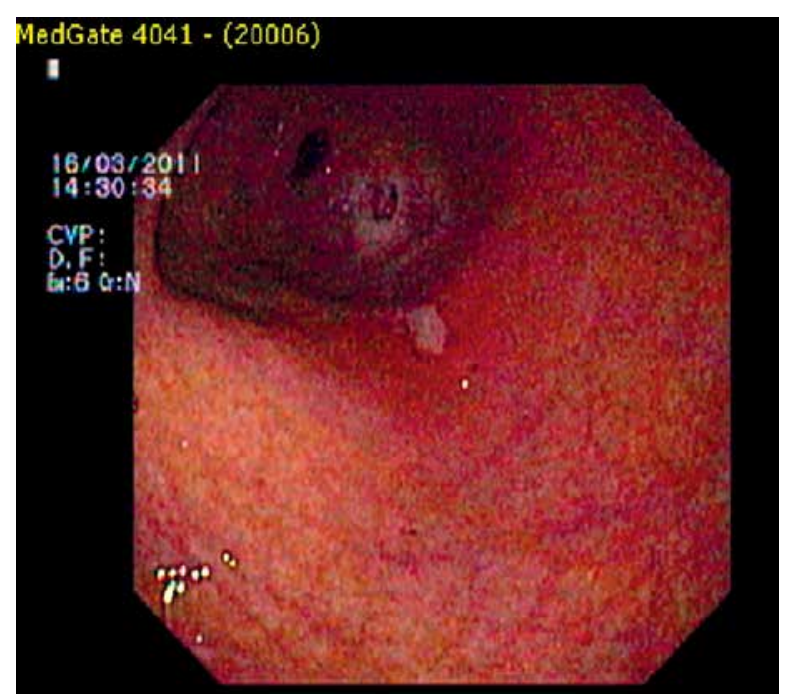

Figure 2. Gastric ulcers due to compression of bezoars revealed after extraction

The aim of the treatment was complete extraction of the bezoar. There are different alternative treatment methods such as liquid diet, prokinetic agents, dissolution of bezoar, and endoscopic or surgical extraction. Coca-Cola may be administered orally or via endoscopy for dissolution of phytobezoars; however, it is not effective on its own and must be given together with a prokinetic agent like metoclopramide [3]. Because trichobezoars are so hard, prokinetic agents and solvents are usually ineffective. Biopsy forceps, alligator forceps, polypectomy snares, and argon plasma coagulation can be used for endoscopic therapy. Small bezoars can be removed by biopsy or alligator forceps while huge bezoars can be extracted with a large polypectomy snare in one or multiple sessions. If it is impossible to extract bezoar completely by endoscopic route or any complication occurs, surgery is the option for further treatment. Complete removal of the bezoar is the goal of surgical treatment, and it can be performed by laparotomy or laparoscopy [4]. Moreover, psychotherapy is recommended. Endoscopic surveillance must be carried out if the disease recurs frequently.

\section{Conflict of interest}

The authors declare no conflict of interest.

\section{References}

1. Mihai C, Mihai B, Drug V, Cijevschi Prelipcean C. Gastric bezoars - diagnostic and therapeutic challenges. J Gastrointest Liver Dis 2013; 22: 111.

2. Iwamuro M, Tanaka S, Shiode J, et al. Clinical characteristics and treatment outcomes of nineteen Japanese patients with gastrointestinal bezoars. Intern Med 2014; 53: 1099-105.

3. Goksugur SB, Karataş Z, Bekdaş M, Dilek M. Dissolution of gastric bezoars using cola. Turk J Gastroenterol 2014; 25: 461-2.

4. Park SE, Ahn JY, Jung HY, et al. Clinical outcomes associated with treatment modalities for gastrointestinal bezoars. Gut Liver 2014; 8: 400-7.

Received: 10.02.2015

Accepted: 10.04.2015 